\title{
Guiding Irregular Nuclear Morphology on Nanopillar Array for Malignancy Differentiation in Tumor cells
}

Yongpeng Zeng, ${ }^{1}$ Yinyin Zhuang, ${ }^{1}$ Aninda Mitra, ${ }^{1}$ Peng Chen, ${ }^{1}$ Isabella Saggio, ${ }^{2,3,4}$ G. V. Shivashankar, ${ }^{5,6}$ Weibo Gao, ${ }^{7,8}$ Wenting Zhao ${ }^{1, *}$

${ }^{1}$ School of Chemical and Biomedical Engineering, Nanyang Technological University, Singapore

${ }^{2}$ Dipartimento di Biologia e Biotecnologie Charles Darwin, Sapienza Università di Roma, Roma, 10 Italy

$11{ }^{3}$ School of Biological Sciences Nanyang Technological University, Singapore

$12 \quad{ }^{4}$ CNR Institute of Molecular Biology and Pathology, Rome, Italy

$13{ }^{5}$ Department of Health Sciences \& Technology (D-HEST), ETH Zurich, Switzerland

$14 \quad{ }^{6}$ Paul Scherrer Institute, Villigen, Switzerland

${ }^{7}$ Division of Physics and Applied Physics, School of Physical and Mathematical Sciences, 16 Nanyang Technological University, Singapore

$17{ }^{8}$ The Photonics Institute and Centre for Disruptive Photonic Technologies, Nanyang Technological 18 University, Singapore 


\section{Abstract}

22 For more than a century, abnormal nuclei in tumor cells, presenting subnuclear invaginations and

23 folds on the nuclear envelope, have been known to be associated with high malignancy and poor

24 prognosis. However, current nuclear morphology analysis focuses on the features of the entire

25 nucleus, overlooking the malignancy-related subnuclear features in nanometer scale. The main

26 technical challenge is to probe such tiny and randomly distributed features inside cells. We here

27 employ nanopillar arrays to guide subnuclear features into ordered patterns enabling their

28 quantification as a strong indicator of cell malignancy. Both breast and liver cancer cells were

29 validated, as well as the quantification of nuclear abnormality heterogeneity. The alterations of

30 subnuclear patterns were also explored as effective readouts for drug treatment. We envision this

31 nanopillar-enabled quantification of subnuclear abnormal features in tumor cells opens a new angle

32 in characterizing malignant cells and studying the unique nuclear biology in cancer.

\section{Teaser}

34 A nanopillar-based assay quantifying the abnormal nuclear morphology in tumor cells at

35 single-cell level. 


\section{Introduction}

Nuclear polymorphism is one characteristic feature widely reported across a variety of cancer types (1). However, clinical grading of tumor cell's nuclei for pathological diagnosis still largely relies on subjective visual inspection of nuclear morphology through optical microscopic imaging (2), which counts on the experience of individual pathologists and inevitably suffers from poor reproducibility and reliability (3). To address this bottleneck, a variety of technologies, including clinical sample labeling (4), computer-aided medical image processing with machine learning or artificial intelligence $(5,6)$, and microfabrication and micropatterning for the whole nucleus guidance $(7,8)$, have been explored for objective and quantitative characterization of the nuclear architecture. Nevertheless, the quantifiable parameters are mainly focused on nuclear size and circularity, uniformity of nuclear chromatin, karyoplasmic ratio, and nucleoli, etc. (5, 9); while the obvious subnuclear abnormalities including folds, invaginations, and inclusions on nuclear envelopes are only evaluated qualitatively due to their random appearance in individual nuclei and huge variations between cells. More critically, a significant technical challenge for their quantification lies in their near-diffraction-limited size (i.e. in tens to hundreds of nanometers scale), which is hard to characterize under optical microscopy.

To probe subcellular properties near or below diffraction-limited size scales, vertically aligned nanopillar arrays have recently been introduced as a powerful tool. They were shown to effectively interrogate plasma membrane properties at the scale of tens to hundreds of nanometers, such as altering the membrane permeability for intracellular delivery of biomolecules (10-13), performing subcellular electroporation and recording intracellular electrophysiology signals (14, 15), manipulating nanoscale membrane topography to recruit endocytic proteins $(16,17)$ and trigger actin polymerization (18), probing cellular mechanics $(19,20)$, and guiding cell adhesion, migration and differentiation $(21,22)$. More interestingly, nanopillars have been reported to reach the nucleus in the intracellular space for probing nuclear deformability and their cytoskeleton regulators in live cells (23), altering the distribution of different lamin proteins along them (24), and rewiring the mechanotransduction from the plasma membrane to the nucleus (24). However, whether nanopillar-induced nuclear deformation correlated with the subnuclear irregularities in cancer cells and how such correlation can be used to assess cancer cell properties have not been explored before.

In this study, we demonstrate a quantitative characterization of subnuclear irregularity in cancer cells using vertically aligned nanopillar arrays. When plated on nanopillars, the subnuclear 
68 features of cancer cells are effectively guided into ordered deformation patterns with readable anisotropicity. Interestingly, the increase of anisotropicity shows an obvious correlation with higher malignancy and faster cell migration. Taking advantage of the single cell resolution with multiple sampling points per nucleus, we evaluate both the heterogeneity in a cancer cell population and the differential response of anti-metastatic drugs between high and low malignant cells.

\section{Results}

\section{Nanopillar guides nuclear shape irregularities in cancer cells}

Differential response of subnuclear morphology to nanopillars among tumor cells with different malignancies was first evaluated (Fig. 1A). Arrays of vertically aligned nanopillars (as shown in the scanning electron micrograph (SEM) (Fig. 1B) were fabricated on transparent quartz coverslip using electron-beam lithography (EBL) and reactive ion etching (RIE). Taking breast cancer cells as a model, we examined two well-known cell lines that exhibit distinct metastatic potential, low malignant MCF-7 and high malignant MDA-MB-231 cells, on both flat and nanopillar arrays. After an overnight culture to allow sufficient generation and stabilization of deformations on nanopillars, the nuclear morphology was visualized via immunostaining of nuclear lamina protein lamin A, a key modulator of nuclear shape in pathogenesis (25). For low malignant MCF-7 cells, they display a smooth nuclear outline on flat substrates without noticeable subnuclear features (representative image shown in Fig. 1C top left panel and more examples shown in fig. S1A left column). In contrast, those cultured on nanopillar arrays generate an ordered array of lamin A rings colocalizing with nanopillar position underneath (Fig. 1C bottom left panel and fig. S1A right column), consistent with the previous report (23). In comparison, the high malignant MDA-MB-231 cells, on the flat surface, show prominent but randomly distributed subnuclear foldings and wrinkles across the nucleus, indicating altered nuclear architecture yet challenging to quantify with irregular shapes (Fig. 1C top right panel and fig. S1B left column). But when cultured on nanopillar arrays, MDA-MB-231 cells exhibit significantly decreased randomness of subnuclear irregular features. Instead, distinct alignment of subnuclear features into line patterns along adjacent nanopillars are clearly observed (Fig. 1C bottom right panel and fig. S1B right column), suggesting a remodeling of the lamin network guided by the local perturbations from nanopillars.

Based on the guided subnuclear features on nanopillars, a quantitative analysis of lamin $\mathrm{A}$ is performed to differentiate MCF-7 and MDA-MB-231 cells. One pronounced difference is the isotropicity of the lamin A network generated patterns on nanopillars: the rings formed in MCF-7 nuclei give isotropic distribution around nanopillars, while the lines aligned across pillar arrays in 
100 MDA-MB-231 cells generate anisotropic intensity profile around each nanopillar with dominant 101 distribution in certain angles. To quantitatively distinguish the isotropic and anisotropic patterns of 102 subnuclear deformation on nanopillars, we removed the nuclear boundary and analyzed specifically 103 the orientation distribution of the lamin A's subnuclear patterns using the OrientationJ (26) plug-in 104 in ImageJ (27), where for each pixel, the angle that aligns dominantly with surrounding signals is 105 calculated and displayed in color hues. The intact images with nuclear boundaries of example cells 106 in Fig. 1C for each condition are shown in fig. S2, where the nuclear boundary of MCF-7 on a flat surface remains uncut as no subnuclear feature is detectable. As shown in Fig. 1D, the isotropic ring deformation of MCF-7 nuclei on nanopillars exhibits a broad and random angle distribution of subnuclear features and thereby generates mixed colors surrounding each nanopillar (Fig. 1D, bottom left). Similarly, the nuclear boundary of MCF-7 on the flat surface also displayed a combination of different angles in individual nuclei, and thus a variety of colors (Fig. 1D, top left). In contrast, the aligned subnuclear deformation in MDA-MB-231 on nanopillars gives rise to a preferred angle across the whole nucleus and thus displaying a prominent yellowish color (Fig. 1D, bottom right). However, such a pillar-guided nuclear pattern in high malignant MDA-MB-231 cells could not be formulated in flat surfaces, which only showed a random distribution of subnuclear groves and invaginations (Fig. 1D, top right). Here, by collecting the angle distribution inside the individual nucleus, we found that the nanopillar array produces a detectable predominant angle for the anisotropic subnuclear deformation in high malignant MDA-MB-231 cells. In contrast, no dominant subnuclear orientation is observed in the low malignant MCF-7 nucleus (Fig. 1E).

For quantitative evaluation of deformation orientations, the anisotropy of the subnuclear features on each nanopillar is further converted into orientation coherency values (i.e., pillar coherency, or p.c. in short), which is ranging from 0 to 1 with 0 representing a completely isotropic pattern (e.g. perfect circle) and 1 refers to an extremely anisotropic pattern (e.g. straight line). Low malignant MCF-7 cells have much lower coherency value (p.c. $=0.27 \pm 0.18, \mathrm{n}=94$ pillars) than high malignant MDA-MB-231 cells (p.c.=0.42 $\pm 0.13, \mathrm{n}=44$ pillars) (Fig. 1F). By averaging the p.c. values of the same cell, we can further obtain an averaged cell coherency value (in short as c.c.) as a single cell readout for cell population analysis. Based on the statistics on individual pillars, we set a coherency value of 0.3 as the threshold to distinguish isotropic and anisotropic subnuclear features in this study. Not surprisingly, MCF-7 contains a higher fraction of ring-deformation cells (c.c. $<0.3$, fraction=0.74 $\pm 0.13, \mathrm{n}=39$ cells) while MDA-MB-231 sample mainly contains line-deformation cells (c.c. $>0.3$, fraction $=0.90 \pm 0.11, \mathrm{n}=24$ cells), as shown in Fig. 1G. It is interesting to note that both low-malignant MCF-7 cells and high-malignant MDA-MB-231 cells 
133 contain a mixed population of low c.c. and high c.c. cells, indicating a possible heterogeneity of

134 canner nuclear properties even within the same cell type. In addition, we validate such

135 nanopillar-guided nuclear deformation in two typical liver cancer cells with divergent invasiveness,

136 highly invasive SK-HEP-1 and non-invasive PLC-PRF-5 (28). By wound healing assay, we found

137 that invasive SK-HEP-1 cells migrated faster than the non-invasive PLC-PRF-5 cells (fig. S3A).

138 Interestingly, when both cell lines were cultured on nanopillar arrays, invasive SK-HEP-1 cells

139 displayed anisotropic line patterns formed in the nuclei, whereas non-invasive PLC-PRF-5 cells

140 exhibited isotropic ring patterns on nanopillar arrays (fig. S3B). The distribution of their p.c. values

141 shows significant difference between these two cell lines (fig. S3C), and the c.c. values effectively

142 differentiate the two cell lines apart (fig. S3D). Taken together, these results confirmed that

143 nanopillar arrays can effectively guide subnuclear morphological irregularities in tumor cells and

144 can generate quantifiable subnuclear readouts for cell malignancy evaluation with single-cell

145 resolution.

In addition, the dynamics of such subnuclear features on nanopillar are further examined in live cells via transient expression of nuclear envelope protein, LAP2 $\square$ fused with green fluorescent protein (LAP2 $\square$-GFP). Strikingly, subnuclear rings in MCF-7 cells are relatively stable on each nanopillar location over 1 hour regardless of the overall movement of the whole nucleus (Fig. 1H upper row), while aligned line patterns of LAP2 $\square$-GFP in MDA-MB-231 cells are able to switch among nearby nanopillar sites following the migration of nucleus (Fig. 1H lower row). It confirms that nanopillars provide persistent guidance on subnuclear deformations and serve as stable sampling points for subnuclear irregularity measurement.

\section{Probe cancer heterogeneity via guided nuclear deformation}

Heterogeneity among cancer cells presents one of the major hurdles in cancer therapy, as cancer metastasis or drug resistance are often observed only in a subset of cells with higher malignancies (29). Taking the advantages of the single-cell resolution of this nanopillar-based nucleus grading system, we were motivated to verify its accuracy in characterizing the heterogeneity of nuclear irregularity in a mixed cell population. First, we generate a series of cell mixtures containing cancer cells of different malignancies by mixing GFP-CAAX tagged low malignant MCF-7 cells with unlabeled high malignant MDA-MB-231 cells in predefined ratios (illustrated in Fig. 2A). A typical microscopy image of a cell mixture containing both cell types on nanopillar arrays is shown in Fig. 2B, where both line deformation in an unlabeled 
plotting the fraction of cells with high deformation coherency $(>0.3)$ on nanopillars against the ratio of unlabeled MDA-MB-231 in the cell mixture, we observed a positive correlation between nanopillar-measured fraction and the portion of high malignant MDA-MB-231 cells in the mixture (Fig. 2C). It indicates that cancer cells in heterogeneous malignancy can be sorted and quantitatively characterized via the nanopillar-guided subnuclear deformation patterns.

In addition, nanopillar-remodeled lamin A patterns are also strongly correlated with cell migration speed, another key indicator of malignant cells. Cell motility of individual cells obtained from live-cell imaging was correlated with their subnuclear deformation patterns on nanopillars for both MCF-7 and MDA-MB-231 cells (Fig. 2D). When pooling together both cells with same nanopillar-remodeled patterns (ring or line separately), we found that lamin A patterns are correlated with cell migration speed by having ring-formation in slow-migrating cells and line-forming within fast-migrating cells despite their cell types, which are clearly shown in cell migration trajectories (Fig. 2E), mean square displacement (MSD) (Fig. 2F), and calculated cell migration rate (Fig. 2G). More interestingly, even among all the low malignant MCF-7 cells, two subpopulations with distinct migrating speed can be differentiated based on nanopillar-guided lamin A patterns (Fig. 2, H-J, and fig. S4). MCF-7 cells with line deformation migrate faster than MCF-7 cells with ring patterns on nanopillars, as similarly measured by longer migration trajectories (Fig. 2H), larger mean square displacement (MSD) (Fig. 2I), and faster cell migration rate (Fig. 2J). It is plausible to speculate that the polarized contractility in fast migrating cells contribute to the high coherency of aligned lamin A patterns. Altogether, the nanostructured platform constitutes an effective technology for probing the heterogeneity in a cancer cell population with single cell resolution.

\section{Evaluate anti-metastatic drug effect via guided nuclear deformation}

Given the ability to identify and characterize high malignant or fast migrating cancer cells in a mixed cell population, we next sought to use the established nanopillar sensing system to evaluate anti-metastatic drugs. More than 90 percent of cancer mortality is caused by cancer metastasis (30). Identifying and developing ant-cancer drugs, particularly those specifically targeting metastasis-prone cells, is an emerging route for new cancer therapy (31). However, the development of anti-metastasis drugs is a daunting yet challenging task as metastasis only develops from a subset of cells and is difficult to evaluate using conventional methods probing the whole cell population, such as western blot, transwell migration, and matrigel invasion assays (32). In comparison, nanopillar-guided subnuclear deformation effectively identifies high malignant or fast 
197 migrating cancer cells with single-cell resolution through lamin A line pattern. Therefore, we

198 hypothesize that the conversion of line patterns of high malignant cells in response to drug

199 treatment can be used to evaluate drug effectiveness against metastasis (as illustrated in Fig. 3A).

200 As a proof of concept, we examined a reported anti-metastatic drug, curcumin $(33,34)$, and

201 compared the deformation changes of MCF-7 and MDA-MB-231 on nanopillars in response to it.

202 As shown in Fig. 3B, the high-malignant MDA-MB-231 cells exhibited significantly less line deformation, while low-malignant MCF7 showed no significant pattern changes. Upon quantification, both decreased pillar coherency values (DMSO, $0.42 \pm 0.13, \mathrm{n}=34$ pillars; curcumin, $0.24 \pm 0.16, \mathrm{n}=52$ pillars) (Fig. 3C) and decreased fractions of line-deformation cells (DMSO, 0.93 $\pm 0.12, n=27$ cells; curcumin, $0.26 \pm 0.27, n=30$ cells) (Fig. 3D) are observed for MDA-MB-231 after curcumin treatment. No significant change in the anisotropy of induced nuclear deformation is found in MCF-7 cells (Fig. 3, C and D). Consistently, MCF-7 cells do not show significant changes in migration speed ( $\mathrm{p}$ value $=0.7440$ ) in response to the curcumin treatment, while MDA-MB-231 cells exhibit a lower migration rate ( $\mathrm{p}$ value=0.0363) (Fig. 3, E and F) after the same treatment. Concentration dependency was further characterized, where both the pillar coherency value and fraction of line-deformation cells responded sensitively to as low as $1 \mu \mathrm{M}$ (fig. S5). Besides curcumin, we also evaluated another reported anti-metastatic drug, haloperidol (35), and obtained similar responses as shown in fig. S6, which further validated that the anisotropy of nanopillar-guided subnuclear deformation can be an effective indicator for anti-metastatic drug evaluation.

\section{Discussion}

Nuclear deformation on nanopillars have been reported earlier in terms of the nuclear stiffness-correlated deformation depth (23) and the local indentation-induced redistribution of lamin proteins (24). However, the reorganization of nuclear morphology, especially the pathologically related subnuclear irregular features, have been overlooked. Our study here demonstrates that, using optimized nanopillar designs, we can effectively guide the subnuclear irregularities into quantifiable patterns reflecting the cell migration ability needed for cancer metastasis. It enables quantitative evaluation of the heterogeneity in a given cell mixture and the cellular response to anti-metastatic drugs, both with single-cell resolution. This establishes an effective strategy to apply nanopillar-based technologies for cancer detection.

It is well known that the nuclear lamina formed by lamin proteins is mechanically sensitive. The extracellular forces can induce differential distribution of lamin A and B as observed in both 
229 micropipette aspiration (36, 37) and nanoneedle perturbations (24). While intracellularly, cytoskeleton-induced nuclear membrane tension has been shown to alter the conformation of lamin $\mathrm{A} / \mathrm{C}(38)$. In the case of nanopillars used here, it not only delivers external force to the nuclear lamina, but also triggers membrane curvature-mediated actin polymerization locally as reported earlier (18). Therefore, the nanopillar platform offers a unique platform to tune the subnuclear pattern of lamin A using different designs of nanopillar geometry. In addition, the formation of isotropic rings or anisotropic lines on nanopillar-deformed cancer nuclei also suggests a coordination of lamin network between adjacent nanopillars. Whether or how it correlated with tumorigenesis and cancer metastasis deserves further studies. correlate with cancer progression, $(39,40)$ but how they are correlated with each other is unclear.

Alterations in both cell migration and nuclear mechanics have been found to closely Recent studies showed that unfolding of nuclear grooves and invaginations correlates with the enhanced cellular contractility via nuclear membrane tension and in turn strongly accelerates cell migration under whole cell compression $(41,42)$. Similarly, we also observed that aligned subnuclear deformation correlated to increased cell migration speed. However, different from the nuclear unfolding with global compression on the entire nucleus, the alignment of subnuclear features on nanopillars are persisting but dynamically adopting different nanopillars along with cell movement. It suggests a different regulation mechanism underlying the local nuclear mechanics in response to cell migration.

The nanopillar assay described in this study enables a quantitative characterization of subnuclear deformation in cancer cells. However, the mechanism of generating subnuclear feature during cancer development is still unknown, which inevitably hinders our interpretation of the nanopillar-induced subnuclear patterns. An in-depth molecular understanding of how such irregularities evolve and whether they associate with specific genetic or metabolic alterations will greatly enrich the nanopillar-based readouts. Moreover, due to the huge morphological variations of cell nuclei among different cancer types (1), the nanopillar dimension optimized in this work may need to be re-evaluated with other cancer types.

\section{Materials and Methods}

\section{Fabrication and characterization of nanopillar arrays}

Nanopillar arrays were fabricated on the quartz chip using electron-beam lithography (EBL) and reactive ion etching (RIE). The quartz chip was cleaned with acetone and isopropyl alcohol and 
260 then spin-coated with $300 \mathrm{~nm}$ polymethylmethacrylate (PMMA) (MicroChem), followed by coating of one thin conductive layer, AR-PC 5090.02 (Allresist). Designed nanoscale patterns were 262 written on the PMMA layer by electron-beam lithography (FEI Helios NanoLab 650) and the 263 PMMA on the exposed areas was subsequently removed in the 3:1

264 isopropanol:methylisobutylketone solution. A Cr mask with $80 \mathrm{~nm}$ thickness was formed via 265 thermal evaporation (UNIVEX 250 Benchtop), followed by lift-off with acetone. Nanopillars were 266 finally revealed after reactive ion etching with a mixture of CF4 and CHF3 (Oxford Plasmalab 80). 267 Characterization of nanopillar dimension was performed using SEM (FEI Helios NanoLab 650) 268 after $10 \mathrm{~nm}$ chromium coating.

269 Cell culture and drug treatment

270 Prior to cell culture, the nanostructured chips were cleaned by air plasma for $10 \mathrm{~min}$ and exposed to UV for $15 \mathrm{~min}$. Subsequently, the nanostructured substrates were coated with fibronectin $(2 \mathrm{ug} / \mathrm{ml}$, 272 Sigma-Aldrich) for 30 minutes at $37^{\circ} \mathrm{C}$. After coating, cell culture was performed on the substrates. 273 All the cell lines used in this work were maintained in the Dulbecco's Modified Eagle Medium (DMEM) (Gibco) supplemented with 10\% fetal bovine serum (FBS) (Life Technologies) and 1\% Penicillin-Streptomycin (Life Technologies) in a standard incubator at $37^{\circ} \mathrm{C}$ with $5 \% \mathrm{CO}$. After overnight incubation and the nuclear deformation was stabilized, the MCF-7 and MDA-MB-231 cells on nanostructures are treated with curcumin (Sigma) or DMSO (Sigma). After 24-hour incubation, the treated cells and untreated cells were fixed with 4\% Paraformaldehyde (PFA) Solution in PBS (Boster biological technology AR1068) for 15 minutes for subsequent immunostaining.

\section{Immunofluorescence staining}

282 Cells cultured on nanopillar arrays were immunostained for lamin A or lamin B1. Cells were washed with pre-warmed PBS two times and fixed with $4 \%$ paraformaldehyde (PFA) in phosphate-buffered saline (PBS) (Boster biological technology AR1068) for 15 minutes. The cells were washed three times with PBS and then permeabilized with 0.5\% Triton X-100 (Sigma) in PBS for 15 minutes. After washing with PBS for three times, samples were blocked using 5\% bovine serum albumin (BSA) (Sigma) in PBS for 1 hour before staining with 1:400 anti-lamin A (Abcam ab26300) and anti-lamin B1 (gift from the Saggio lab in Sapienza University of Rome). Samples were washed three times with PBS and stained with the secondary antibody, Chicken anti-Rabbit 
290 IgG $(\mathrm{H}+\mathrm{L})$ Cross-Adsorbed Secondary Antibody, Alexa Fluor 488 (Invitrogen A21441), 1:500 in

291 staining buffer for 1 hour under room temperature.

292 Confocal imaging and live cell tracking

293 Imaging of the fluorescently labeled cells on nanopillar arrays was performed using laser scanning 294 confocal microscopy (Zeiss LSM 800 with Airyscan). In particular, a Plan-Apochromat 100x/1.4 295 oil objective was used. During imaging, fixed cells were maintained in PBS. Z stack images were 296 acquired with $500 \mathrm{~nm}$ distance between each frame. Live cell imaging and the subsequent

297 fluorescence imaging was performed using a spinning disc confocal microscope (SDC) that is built 298 around a Nikon Ti2 inverted microscope equipped with a Yokogawa CSU-W1 confocal spinning 299 head, a Plan-Apo objective (100x1.45-NA), a back-illuminated sCMOS camera (Orca-Fusion; 300 Hamamatsu). Excitation light was provided by 488-nm/150mW (Vortran) (for GFP), and all image 301 acquisition and processing were controlled by MetaMorph (Molecular Device) software. The 302 migration of individual cells was manually tracked using imageJ, and their migratory behavior was 303 characterized using the method developed by a previous work (43).

\section{Transfection}

305 For plasmid transfection in cancer cells, $1 \mu \mathrm{g}$ plasmid was mixed with $1.5 \mu$ Lipofectamine 3000 (Life Technologies) and $2 \mu \mathrm{l} 3000$ reagent (Life Technologies) in Opti-MEM (Gibco) and 307 incubated for 20 mins at room temperature. Before the addition of the transfection mixture, cancer 308 cells were starved with the Opti-MEM (Gibco) medium for $30 \mathrm{mins}$ at $37^{\circ} \mathrm{C}$. After 4 hours of 309 incubation, the Opti-MEM (Gibco) medium was replaced with regular culture medium and the cells were allowed to recover overnight before cell sorting or live cell imaging.

\section{$311 \quad$ Fluorescence $\square$ activated cell sorting}

312 Cells were sorted by using the BD FACS Aria II, and gating was done using the BD FACSDiva ${ }^{\mathrm{TM}}$ 313 software (Becton, Dickinson Biosciences). Dead cells were excluded from analysis on the basis of 314 FSC/SSC; cell aggregates or small debris were excluded from analysis on the basis of side scatter 315 (measuring cell granularity) and forward scatter (measuring cell size); lastly, GFP positive cells 316 were sorted on the basis of fluorescence intensity.

\section{Wound healing assay}


318 Cells were maintained in $35 \mathrm{~mm}$ dishes for each cell line until approximately $90 \%$ confluent.

319 Scratch was made in the confluent monolayer of cells with a sterile 200- $\mu 1$ pipette tip, and fresh

320 culture medium was replaced. Brightfield microscopic pictures were taken of the same field at 24

321 hours. Migration rate was measured by quantifying the closure area within the same time frame

322 using ImageJ.

\section{Statistical analysis}

324 Welch's t tests (unpaired, 2 tailed, not assuming equal SD) were used to evaluate the significance.

325 All tests were performed using Prism (GraphPad Software). Data are presented as mean \pm SEM or 326 mean \pm SD as stated in the figure captions. All experiments were repeated at least twice, unless 327 otherwise explicitly stated in the figure captions. 


\section{References}

1. D. Zink, A. H. Fischer, J. A. Nickerson, Nuclear structure in cancer cells. Nature Reviews Cancer 4, 677-687 (2004).

2. J. I. de Las Heras, E. C. Schirmer, The nuclear envelope and cancer: a diagnostic perspective and historical overview. Advances in experimental medicine and biology 773, 5-26 (2014).

3. J. P. Sloane et al., Consistency achieved by 23 European pathologists from 12 countries in diagnosing breast disease and reporting prognostic features of carcinomas. European Commission Working Group on Breast Screening Pathology. Virchows Archiv : an international journal of pathology 434, 3-10 (1999).

4. G. Bussolati et al., "To be or not to be in a good shape": diagnostic and clinical value of nuclear shape irregularities in thyroid and breast cancer. Advances in experimental medicine and biology 773, 101-121 (2014).

5. A. Das, M. S. Nair, S. D. Peter, Computer-Aided Histopathological Image Analysis Techniques for Automated Nuclear Atypia Scoring of Breast Cancer: a Review. Journal of digital imaging 33, 1091-1121 (2020).

6. A. Radhakrishnan, K. Damodaran, A. C. Soylemezoglu, C. Uhler, G. V. Shivashankar, Machine Learning for Nuclear Mechano-Morphometric Biomarkers in Cancer Diagnosis. Scientific Reports 7, 17946 (2017).

7. Z. Pan et al., Control of cell nucleus shapes via micropillar patterns. Biomaterials 33, 1730-1735 (2012).

8. F. Badique et al., Directing nuclear deformation on micropillared surfaces by substrate geometry and cytoskeleton organization. Biomaterials 34, 2991-3001 (2013).

9. G. Kristiansen, Next-generation nuclear morphology to grade solid tumours. The Lancet Oncology 19, 275-277 (2018).

10. C. Chiappini et al., Biodegradable silicon nanoneedles delivering nucleic acids intracellularly induce localized in vivo neovascularization. Nature Materials 14, 532-539 (2015).

11. R. Elnathan et al., Maximizing Transfection Efficiency of Vertically Aligned Silicon Nanowire Arrays. Advanced Functional Materials 25, 7215-7225 (2015).

12. J. J. VanDersarl, A. M. Xu, N. A. Melosh, Nanostraws for direct fluidic intracellular access. Nano letters 12, 3881-3886 (2012).

13. A. K. Shalek et al., Vertical silicon nanowires as a universal platform for delivering biomolecules into living cells. 107, 1870-1875 (2010).

14. C. Xie, Z. Lin, L. Hanson, Y. Cui, B. Cui, Intracellular recording of action potentials by nanopillar electroporation. Nature Nanotechnology 7, 185-190 (2012).

15. X. Duan, T. M. Fu, J. Liu, C. M. Lieber, Nanoelectronics-biology frontier: From nanoscopic probes for action potential recording in live cells to three-dimensional cyborg tissues. Nano today 8, 351-373 (2013).

16. W. Zhao et al., Nanoscale manipulation of membrane curvature for probing endocytosis in live cells. Nature Nanotechnology 12, 750-756 (2017).

17. H. Y. Lou, W. Zhao, Y. Zeng, B. Cui, The Role of Membrane Curvature in Nanoscale Topography-Induced Intracellular Signaling. Accounts of chemical research 51, 1046-1053 (2018).

18. H.-Y. Lou et al., Membrane curvature underlies actin reorganization in response to nanoscale surface topography. 116, 23143-23151 (2019).

19. J. L. Tan et al., Cells lying on a bed of microneedles: An approach to isolate mechanical force. 100, 1484-1489 (2003).

20. J.-Y. Shiu, L. Aires, Z. Lin, V. Vogel, Nanopillar force measurements reveal actin-cap-mediated YAP mechanotransduction. Nature Cell Biology 20, 262-271 (2018). 
21. B. K. K. Teo et al., Nanotopography Modulates Mechanotransduction of Stem Cells and Induces Differentiation through Focal Adhesion Kinase. ACS Nano 7, 4785-4798 (2013).

22. S. Bonde et al., Tuning InAs Nanowire Density for HEK293 Cell Viability, Adhesion, and Morphology: Perspectives for Nanowire-Based Biosensors. ACS Applied Materials \& Interfaces 5, 10510-10519 (2013).

23. L. Hanson et al., Vertical nanopillars for in situ probing of nuclear mechanics in adherent cells. Nat Nanotechnol 10, 554-562 (2015).

24. C. S. Hansel et al., Nanoneedle-Mediated Stimulation of Cell Mechanotransduction Machinery. ACS Nano 13, 2913-2926 (2019).

25. P. Isermann, J. Lammerding, Nuclear mechanics and mechanotransduction in health and disease. Current biology : CB 23, R1113-1121 (2013).

26. E. Fonck et al., Effect of aging on elastin functionality in human cerebral arteries. Stroke 40, 2552-2556 (2009).

27. J. Schindelin et al., Fiji: an open-source platform for biological-image analysis. Nature Methods 9, 676-682 (2012).

28. B. Rani et al., Galunisertib suppresses the staminal phenotype in hepatocellular carcinoma by modulating CD44 expression. Cell Death \& Disease 9, 373 (2018).

29. I. Dagogo-Jack, A. T. Shaw, Tumour heterogeneity and resistance to cancer therapies. Nature reviews. Clinical oncology 15, 81-94 (2018).

30. C. L. Chaffer, R. A. Weinberg, A perspective on cancer cell metastasis. Science (New York, N.Y.) 331, 1559-1564 (2011).

31. R. L. Anderson et al., A framework for the development of effective anti-metastatic agents. Nature Reviews Clinical Oncology 16, 185-204 (2019).

32. A. Valster et al., Cell migration and invasion assays. Methods (San Diego, Calif.) 37, 208-215 (2005).

33. L. G. Menon, R. Kuttan, G. Kuttan, Anti-metastatic activity of curcumin and catechin. Cancer letters 141, 159-165 (1999).

34. R. A. Sharma et al., Pharmacodynamic and pharmacokinetic study of oral Curcuma extract in patients with colorectal cancer. Clinical cancer research : an official journal of the American Association for Cancer Research 7, 1894-1900 (2001).

35. W. Zhao et al., Candidate Antimetastasis Drugs Suppress the Metastatic Capacity of Breast Cancer Cells by Reducing Membrane Fluidity. Cancer research 76, 2037-2049 (2016).

36. J. D. Pajerowski, K. N. Dahl, F. L. Zhong, P. J. Sammak, D. E. Discher, Physical plasticity of the nucleus in stem cell differentiation. 104, 15619-15624 (2007).

37. Y. Xia et al., Rescue of DNA damage after constricted migration reveals a mechano-regulated threshold for cell cycle. Journal of Cell Biology 218, 2545-2563 (2019).

38. T. O. Ihalainen et al., Differential basal-to-apical accessibility of lamin A/C epitopes in the nuclear lamina regulated by changes in cytoskeletal tension. Nature Materials 14, 1252-1261 (2015).

39. C. Denais, J. Lammerding, Nuclear mechanics in cancer. Advances in experimental medicine and biology 773, 435-470 (2014).

40. C. H. Stuelten, C. A. Parent, D. J. Montell, Cell motility in cancer invasion and metastasis: insights from simple model organisms. Nature Reviews Cancer 18, 296-312 (2018).

41. A. J. Lomakin et al., The nucleus acts as a ruler tailoring cell responses to spatial constraints. Science (New York, N.Y.) 370, (2020).

42. V. Venturini et al., The nucleus measures shape changes for cellular proprioception to control dynamic cell behavior. Science (New York, N.Y.) 370, (2020).

43. P. H. Wu, A. Giri, D. Wirtz, Statistical analysis of cell migration in 3D using the anisotropic persistent random walk model. Nature protocols 10, 517-527 (2015). 


\section{Acknowledgments}

129 Funding: This work is supported by the Singapore Ministry of Education (MOE) (W. Zhao,

130 RG145/18, RG112/20, and MOE-MOET32020-0001), the Singapore National Research

431 Foundation (W. Zhao, NRF2019-NRF-ISF003-3292), the NTU Start-up Grant (W. Zhao),

332 NTU-NNI Neurotechnology Fellowship (W. Zhao), and AIRC IG-24614 and Sapienza

433 AR1181642EE61111 (I. Saggio).

134 Author contribution: Y.P.Z. and W.Z. conceived the idea and designed the experiments. Y.P.Z. 135 and Y.Y.Z. performed most of the experiments and data analysis. Y.P.Z. and W.G. performed 336 fabrication and SEM experiments. P.C., I.S. and G.V.S. provided material and experimental 437 supports. Y.P.Z. and W.Z. drafted the manuscript. W.G., P.C., I.S. and G.V.S. edited the 438 manuscript. All of the authors discussed and commented on the manuscript.

439 Competing interests: Y.P.Z., A.M. and W.Z. are inventors on a pending patent related to this 440 work filed by Nanyang Technological University (no. PCT/SG2021/050687, filed on 10

141 November 2021). The authors declare that they have no other competing interests.

442 Data and materials availability: All data needed to evaluate the conclusions in the paper are 443 present in the paper and/or the Supplementary Materials. 
444 Figures and Tables

A

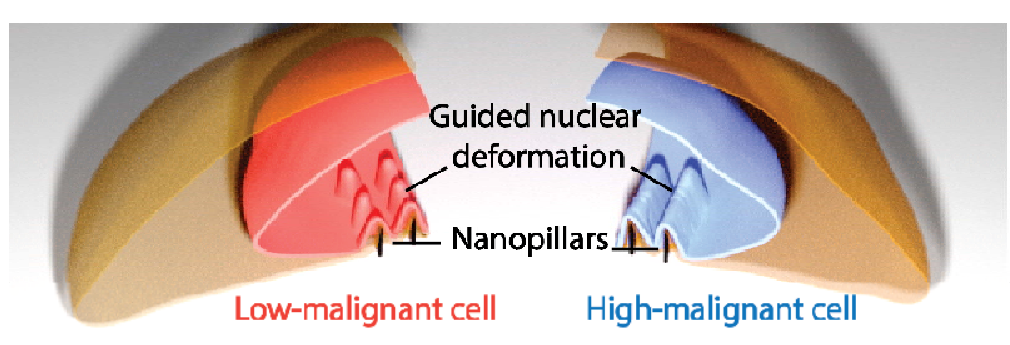

C

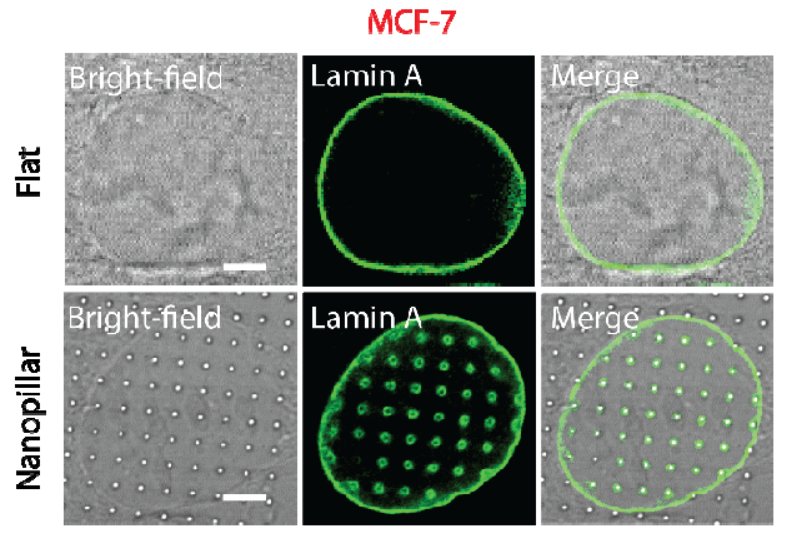

E

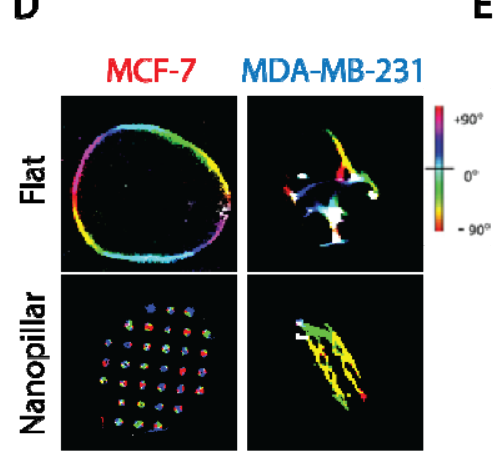

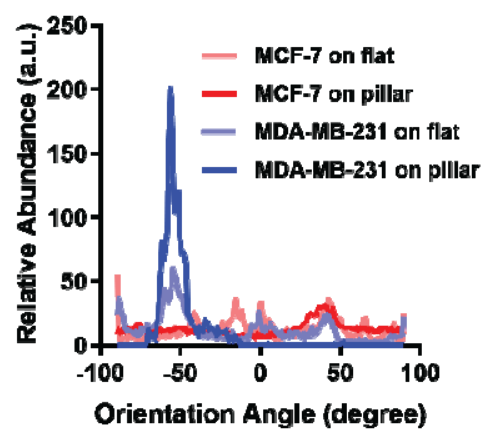

$\mathbf{F}$
B

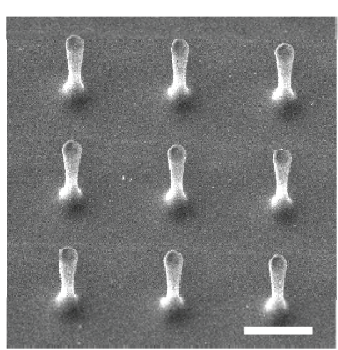

MDA-MB-231
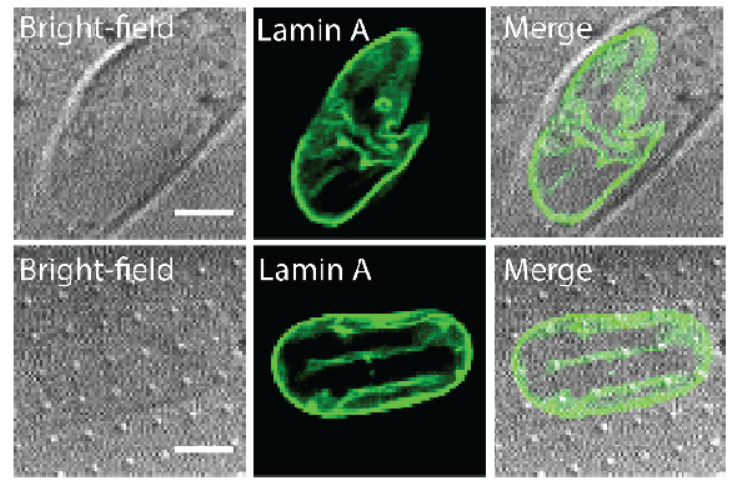

G $\square$ c.c. $<0.3$ (Ring)
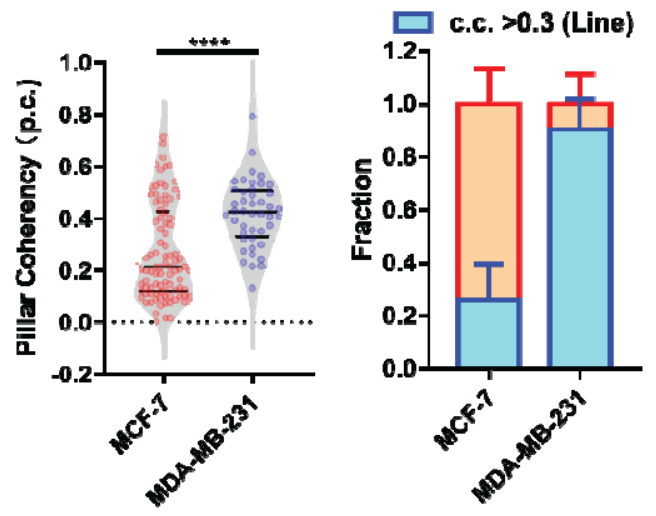

H

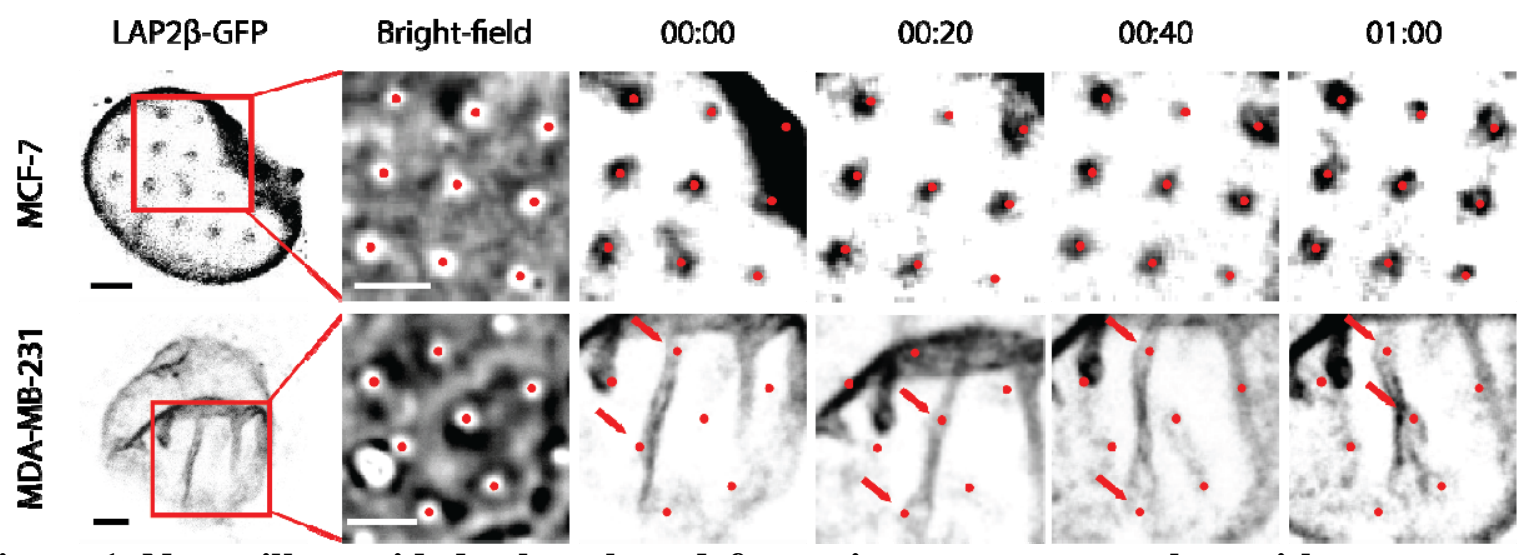

Figure 1. Nanopillar-guided subnuclear deformation patterns correlate with cancer

malignancy. (A) Schematics of different patterns generated by nanopillar-guided nuclear shape deformation in cancer cells with varying malignancies. (B) SEM of nanopillar arrays. Scale bar, 2 $\mu \mathrm{m}$. (C) Nuclear morphology of MCF-7 cells and MDA-MB-231 cells on a flat surface. Scale bars, $5 \mu \mathrm{m}$. (D) Orientation of nuclear shape irregularities and nanopillar-guided nuclear features 
451 in MDA-MB-231 cells and MCF-7 cells. (E) Comparison of orientation distribution of nuclear

452 shape irregularities and nanopillar-guided nuclear features in MDA-MB-231 cells and MCF-7

453 cells. (F) Anisotropy measurement of the nanopillar-guided nuclear deformation in MCF-7 cells

$454(\mathrm{~N}=94$ pillars $)$ and MDA-MB-231 cells $(\mathrm{N}=44$ pillars). (G) Fraction of ring deformation and

455 line deformation in MCF-7 cells $(\mathrm{N}=39$ cells $)$ and MDA-MB-231 cells $(\mathrm{N}=24$ cells). Ring

456 deformation is defined by c.c. $<0.3$ whereas ring deformation is defined by c.c. $>0.3$. (H)

457 Dynamics of nanopillar-guided nuclear features in MCF-7 and MDA-MB-231 cells for one hour.

458 Red dots indicate nanopillar locations. Red arrows in the bottom row refer to the nanopillars that

459 guide the nuclear grooves. Scale bars, $3 \mu \mathrm{m}$. Statistical significance of the two groups was

460 compared using an unpaired t test with Welch's correction, $\mathrm{p}$-value: $* * * *<0.0001$. 
A

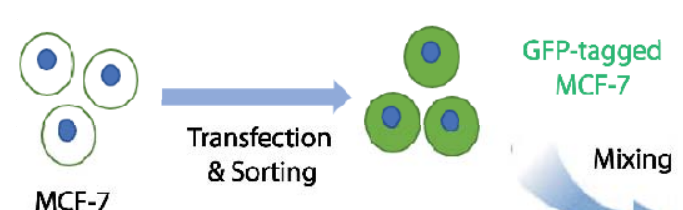

B
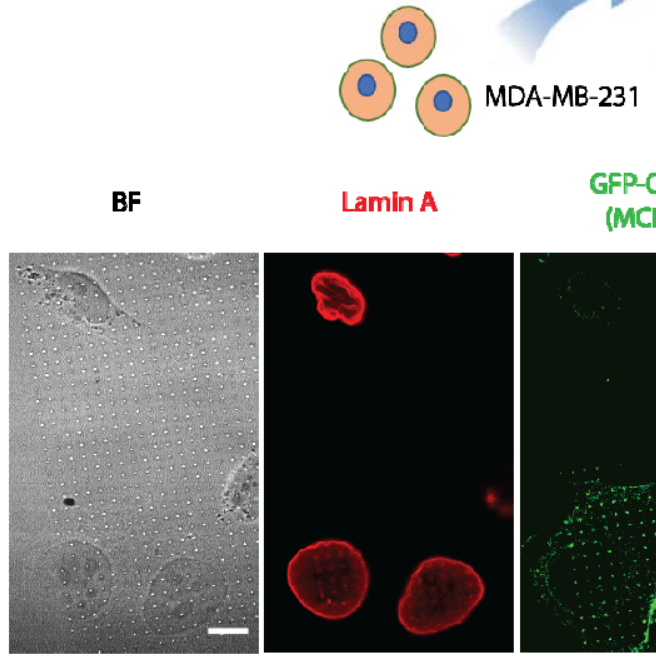

$\operatorname{Lamin} \mathbf{A}$
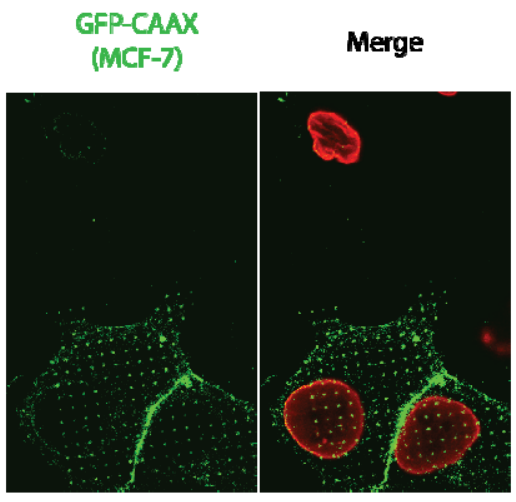

D

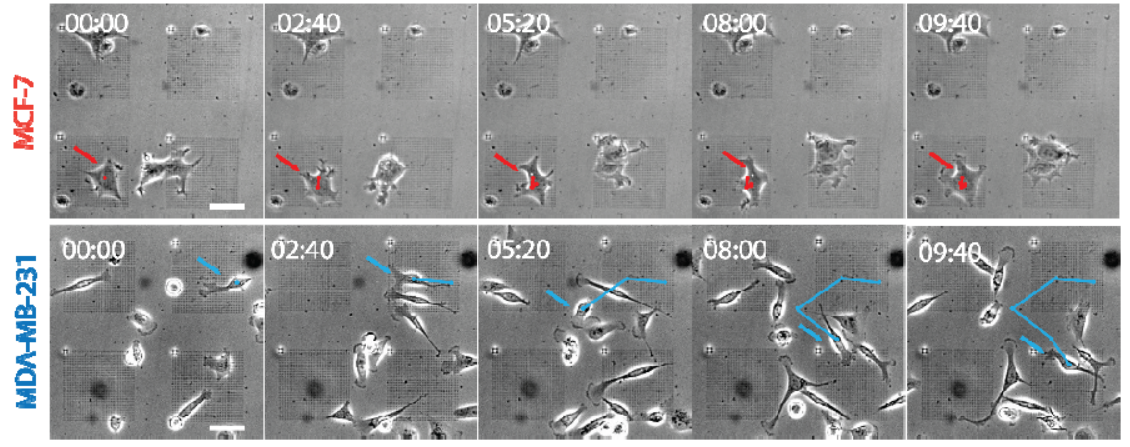

E

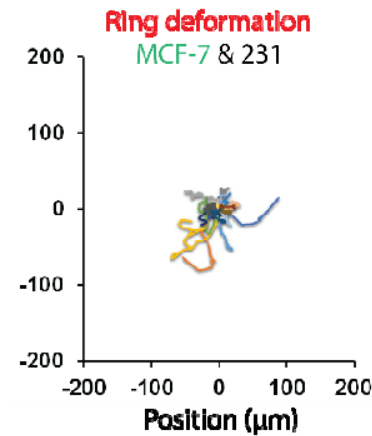

H

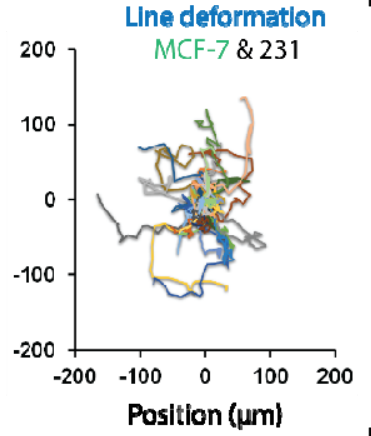

$\mathbf{F}$

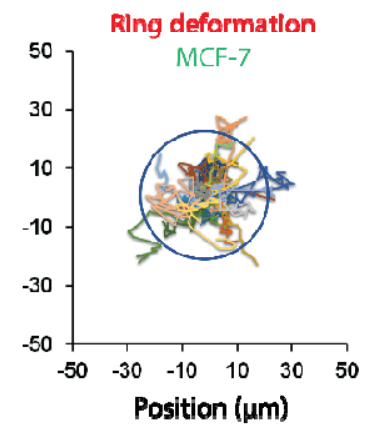

LIne deformatlon

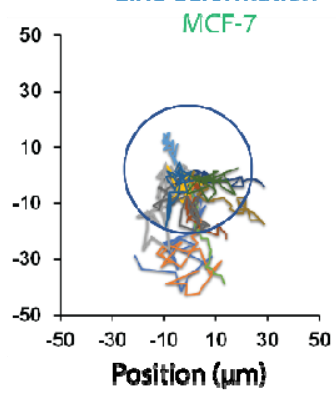

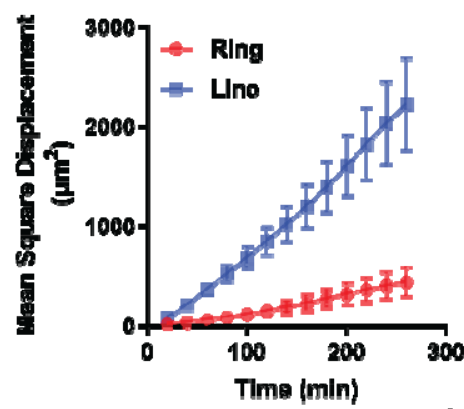

G
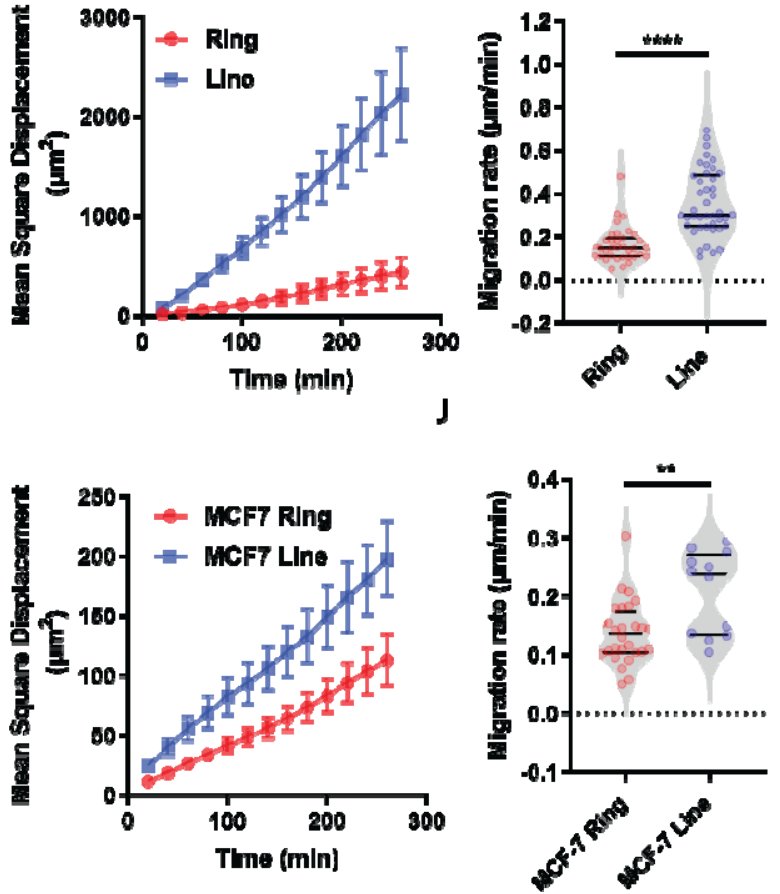

Ring deformation

LIne deformation

C

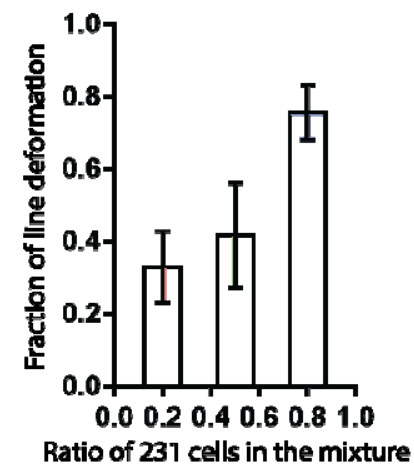


462 Figure 2. Probing cancer heterogeneity via nanopillar-induced subnuclear deformation. (A)

163 Methodology for using nanopillar arrays to probe cancer heterogeneity. (B) MDA-MB-231 cell

464 and GFP-tagged MCF-7 cells showing different nuclear deformation patterns on the same 465 substrate. Scale bar $10 \mu \mathrm{m}$. (C) Correlation between fraction of cells showing line-like guided nuclear deformation and the ratio of MDA-MB-231 cells to MCF-7 cells. (D) Brightfield images of MCF-7 cells and MDA-MB-231 cells migrating on nanopillar arrays over time and the fluorescent images showing deformation patterns of cells at the last time point. Scale bars for left figures, $50 \mu \mathrm{m}$. Scale bars for right figures, $10 \mu \mathrm{m}$. (E) Migration trajectories of cells showing different nuclear deformation patterns on nanopillars (Ring: $\mathrm{N}=35$ cells; Line: $\mathrm{N}=42$ cells). (F) MSD measurement of cells showing different nuclear deformation patterns. (G) Comparison of migration rate of cells showing varying nuclear deformation patterns. (H) Migration trajectories of MCF-7 cells showing different nuclear deformation patterns on nanopillars (MCF7 ring: $\mathrm{N}=28$ cells; MCF7 line: $\mathrm{N}=12$ cells). Two blue circles with the same diameter are centered with the origin to show that MCF-7 cells with line deformation on nanopillars tend to migrate faster than those showing ring deformation. (I) MSD measurement of MCF-7 cells showing different nuclear deformation patterns. (J) Comparison of migration rate of MCF-7 cells showing varying nuclear deformation patterns. Statistical significance of migration rate measurement under different $480 \quad 0.01$ 
A

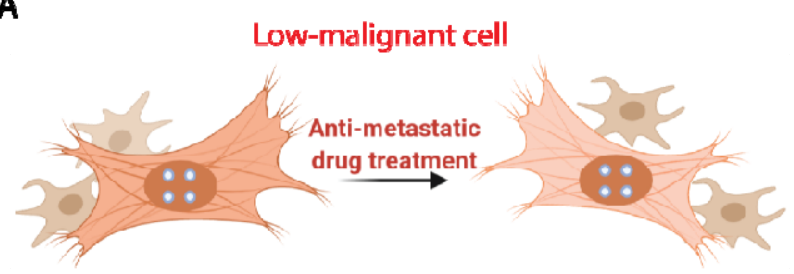

B

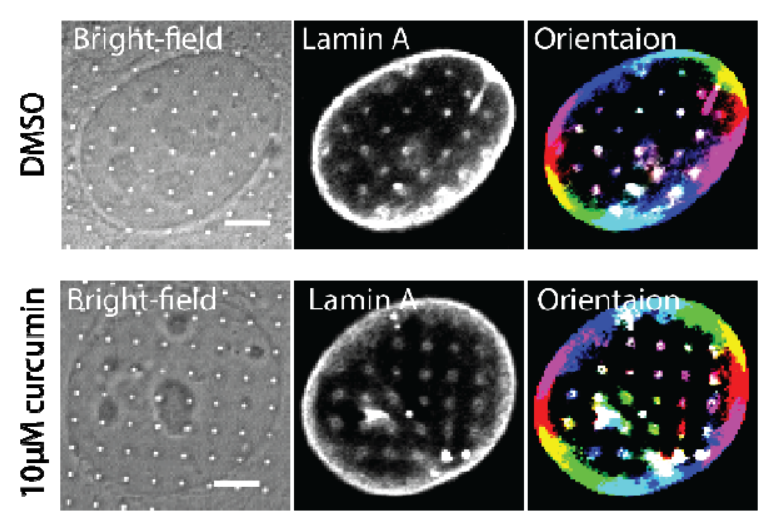

C

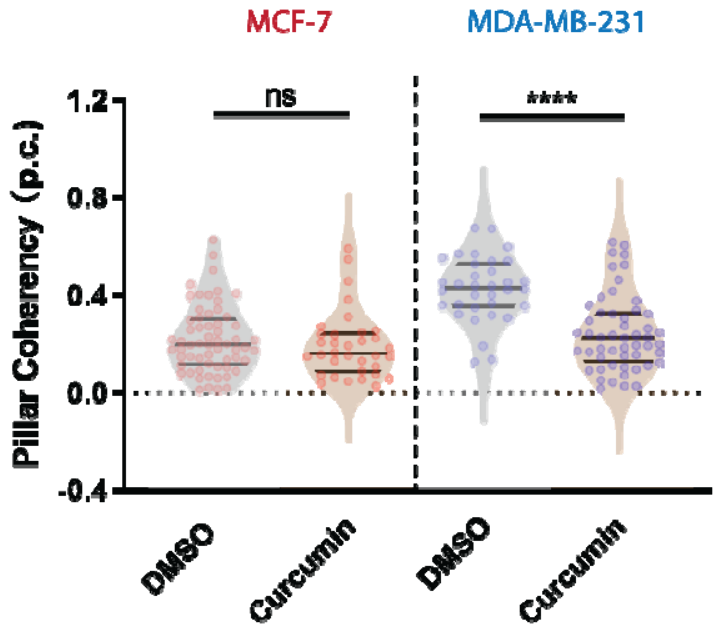

E

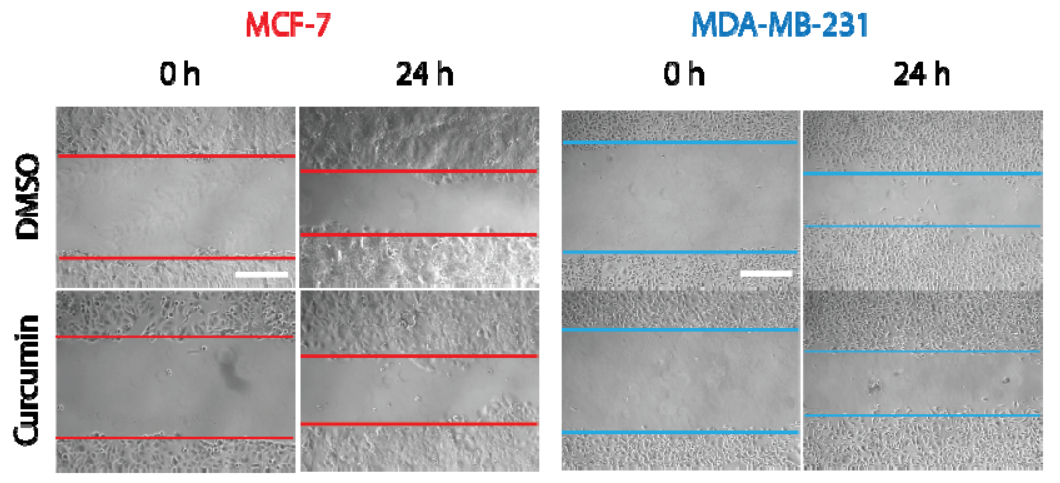

High-malignant cell

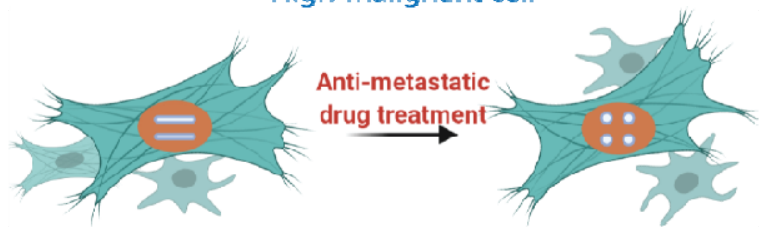

MDA-MB-231
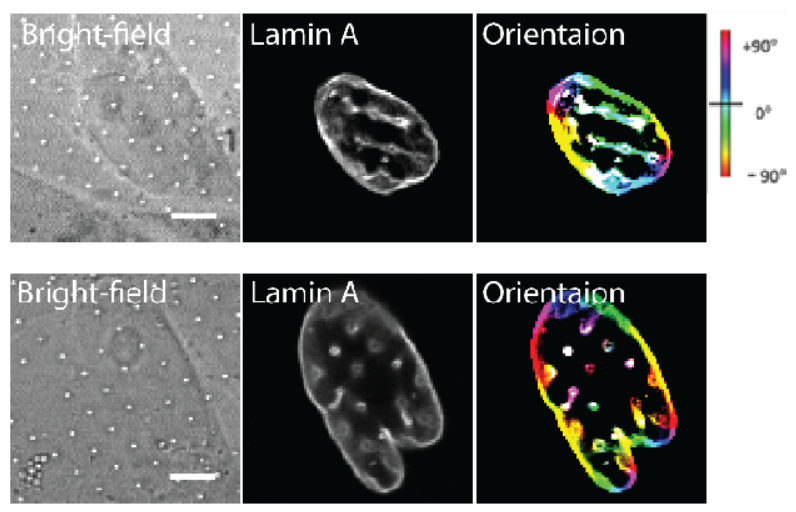

D

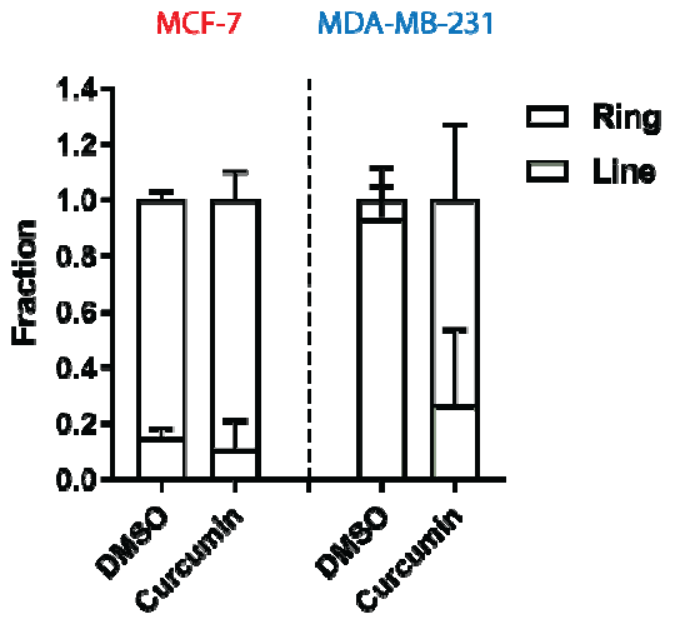

$\mathbf{F}$

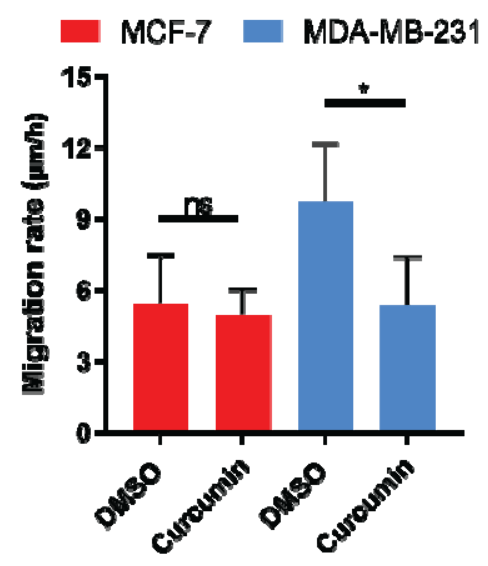

Figure 3. Evaluating anti-metastatic drug effects via nanopillar-induced subnuclear deformation. (A) Characterize response of cancer cells with varying malignancies to anti-metastatic drug treatment using deformation anisotropicity. (B) Nuclear deformation patterns and their orientation of MCF-7 cells and MDA-MB-231 cells on nanopillar arrays with or without 
486 curcumin treatment. Scale bar, $5 \mu \mathrm{m}$. (C) Anisotropy measurement of nanopillar-guided nuclear 187 features in MCF-7 cells and MDA-MB-231 cells with or without curcumin treatment (MCF-7:

$488 \mathrm{~N}=61$ pillars (DMSO); $\mathrm{N}=34$ pillars (curcumin); MDA-MB-231: $\mathrm{N}=34$ pillars (DMSO); $\mathrm{N}=52$ 489 pillars (curcumin).). (D) Fraction of ring deformation and line deformation in MDA-MB-231 cells 490 and MCF-7 cells on nanopillar arrays with or without curcumin treatment (MCF-7: N= 35 cells 191 (DMSO); $\mathrm{N}=21$ cells (curcumin); MDA-MB-231: $\mathrm{N}=27$ cells (DMSO); $\mathrm{N}=30$ cells (curcumin).). 492 Error bars represent SD. (E) Wound healing assay of MCF-7 cells and MDA-MB-231 cells with 493 or without curcumin treatment for $24 \mathrm{~h}$. Scale bars, $200 \mu \mathrm{m}$. (F) Migration rate of MCF-7 cells and 194 MDA-MB-231 cells under different conditions was measured using wound healing assay ( $\mathrm{N}=3$ 495 batches). Statistical significance of measurement for coherency and migration rate under different 196 conditions was evaluated by an unpaired t-test with Welch's correction. ****P $<0.0001$; *P < $497 \quad 0.05 ; \mathrm{ns}>0.05$. 